\title{
Commentary
}

\section{Sex hormones and ApoE4: Beyond steroids}

\author{
Richard L. Bowen* and Christopher W. Gregory \\ Voyager Pharmaceutical Corporation, 8540 Colonnade Center Drive, Raleigh, NC 27615, USA
}

The accompanying paper by Burkhardt and colleagues [2] highlights the complexity of the estrogen/Alzheimer's disease (AD) connection. One possible explanation of these interesting results is that there is a link between particular estrogen receptor polymorphisms and apolipoprotein E (ApoE) polymorphisms. While there are conflicting reports in the literature, several studies have suggested an increased risk of AD in women with particular estrogen receptor polymorphisms [1]. There is a well established physiologic relationship between estrogen and apolipoproteins due to the fact that estrogen is a reproductive steroid hormone formed from cholesterol, the primary source of which is ApoE. Considering the importance of estrogen in regulation of reproductive function, it is easy to imagine that individuals with ApoE4 might be more fertile if they possessed a particular estrogen receptor polymorphism.

Alternatively, the ApoE polymorphism dependent differential effect of estrogen on cognition might be explained by the finding that there appears to be a "critical period" during which hormone replacement therapy (HRT) must be initiated in order to have a protective effect on cognition [4]. One possible explanation for the finding that estrogen exposure had no effect on cognition in individuals possessing an ApoE4 gene relates to this "critical period" hypothesis. Since published data suggests that the possession of an ApoE4 allele is associated with an earlier onset of cognitive decline [3], it may be that the "critical period" for patients positive for ApoE4 begins before it does for ApoE4 nega-

\footnotetext{
${ }^{*}$ Corresponding author. E-mail: rbowenmd@voyagerpharma. com.
}

tive individuals. Therefore, even though the duration of estrogen exposure was similar for subjects in this study, for those positive for ApoE4 the "critical period" may have already passed when they began therapy. In this situation, rather than excluding HRT as a potential method of preserving cognition in ApoE4 patients, they should be considered for earlier initiation of HRT.

Unfortunately, due to the Women's Health Initiative studies thus far published, this paper notwithstanding, it is unlikely that clinicians will recommend HRT as a means of preventing disease whether it be Alzheimer's disease, arterial disease, or even osteoporosis. Rather they will reserve HRT for those patients who need it to treat the unpleasant symptomatic effects of menopause. While this study may not have direct implications on current clinical practice, it may well provide clues into the mechanism by which ApoE4 confers an increased risk of AD. Stratifying the estrogen treated ApoE4 patients into quartiles (non-responders vs. responders) and looking for differences in estrogen receptor polymorphisms between these groups might be a first step. It is also important to keep in mind that the hypothalamic-pituitary-gonadal (HPG) axis is a complex endocrine feedback loop involving gonadotropin releasing hormone, leuteinizing hormone, follicle stimulating hormone, activin, inhibin, and follistatin, as well as the sex steroids. Just as with estrogen, the receptors for many of these hormones are expressed in multiple non-reproductive tissues. By definition, in any hormonal "feedback loop" altering the serum concentrations of any one hormone effects the serum concentrations of numerous others. Therefore, identifying the specific mechanism(s) by which a treatment such as HRT alters the clinical course of a disease becomes extremely complex. Effects seen with HRT may be 
due to the effect of estrogen not only on the serum concentrations of other hormones but also effects on expression levels of their receptors. Therefore, while it remains clear that the age-related changes in reproductive hormones play a role in numerous diseases of aging including $\mathrm{AD}$, further research into the mechanism(s) by which this occurs should include a thorough study of all the hormones and receptors of the HPG axis.

\section{References}

[1] M. Brandi, L. Becherini, L. Gennari, M. Racchi, A. Bianchetti, B. Nacmias, S. Sorbi, P. Mecocci, U. Senin and S. Govoni, As- sociation of the estrogen receptor alpha gene polymorphisms with sporadic Alzheimer's disease, Biochemical and Biophysical Research Communications 265 (1999), 335-338.

[2] M.S. Burkhardt, J.K. Foster, S.M. Laws, L.D. Baker, S. Craft, S.E. Gandy, B.G.A. Stuckey, R. Clarnette, D. Nolan, B. Hewson-Bower and R.N. Martins, Oestrogen replacement therapy may improve memory functioning in the absence of ApoE $\varepsilon 4$, Journal of Alzheimer's Disease 6(3) (2004), 221-228.

[3] E. Ghebremedhin, C. Schultz, E. Braak and H. Braak, High frequency of apolipoprotein E epsilon4 allele in young individuals with very mild Alzheimer's disease-related neurofibrillary changes, Experimental Neurology 153 (1998), 152-155.

[4] R. B. Gibbs and R. Gabor, Estrogen and cognition: applying preclinical findings to clinical perspectives, Journal of Neuroscience Research 74 (2003), 637-643. 